\title{
UsaLab: the Experience of a Usability Lab from the Mexican Perspective
}

\author{
Mario A Moreno Rocha \\ Universidad Tecnológica de la Mixteca \\ Km 2.5 Carretera a Acatlima \\ Huajuapan de León, Oaxaca CP 69000, Mexico \\ + 5295320399 ext 200 \\ mmoreno@mixteco.utm.mx
}

\author{
Dayfel Hernández Martínez \\ UsaLab \\ Km 2.5 Carretera a Acatlima \\ Huajuapan de León, Oaxaca CP 69000, Mexico \\ + 52953200214 ext 200 \\ dayfel@mixteco.utm.mx
}

\begin{abstract}
This paper presents the knowledge and lessons learned from our experience running the first usability lab in Mexico, originated from the academia and now moving into servicing the industry. It also describes the HCI research made in our university, our current projects and future direction, from a Mexican perspective.
\end{abstract}

\section{Categories and Subject Descriptors}

K.1 The Computer Industry. K.3 Computers and Education: K.3.1 Computer Uses in Education. K.7 The Computing Profession: K.7.3 Testing, Certification and Licensing.

\section{General Terms}

Management, Human Factors, Languages.

\section{Keywords}

HCI, Mexico, UsaLab, usability testing, UTM

\section{INTRODUCTION}

The academic research and HCI practice in Mexico is a novel activity, breaking into the establishment and trying to find its own space. It's a collective effort from both academic and industrial communities, developing exciting research and spreading the word of HCI and usability everywhere. More and more people are getting involved in such efforts and interesting results are being achieved, positioning our country close to the lead in the Latin American HCI community.

The Universidad Tecnológica de la Mixteca (UTM) is located in the southern Mexican state of Oaxaca, a region typically known both as a famous traditional tourist destination, but also as the least developed state in the country. Ours is a public university of technology rising to create development through excellence in academy, scientific research and social improvement. The study and practice of Human ComputerInteraction is one of the key issues to reach our objectives.

HCI started humbly in 2001 in our university, with a Ben Shneiderman's book in our university's library, but with great interest. Years later, our university team beat the University of Maryland's at the SIGCHI Student Design Competition Finals in 2007.

The rapid progress of HCI in our university was achieved in three fronts, described briefly below.

\subsection{Research}

We created a research group with general interest in software engineering, the CASI, in which the research line of Human Computer-Interaction was formalised. Our interest was to create research in areas such as GUIs for people with disabilities, mobile interfaces and developments on localisation and transcultural usability.

Since then, our research group has published multiple papers, made national and international presentations, as well as organised workshops and tutorials. Participation includes SIGCHI, HCI International, IWIPS, Interact, British HCI, Mensch \& Computer (Germany), Interfaces (Spain), MexIHC (Mexico), Interacción (Spain), CLHIC (Latin America).

\subsection{Academic Affairs}

We have just added the Human Computer-Interaction subject into the new curricula for the Computing Engineering Bachelor Degree [1], and it is part of the Informatics Bachelor Degree in our university's system for years now. Our new graduate studies, the MSc in Interactive Systems feature three different modules on $\mathrm{HCI}$, being the graduate studies with more $\mathrm{HCI}$ studies in its curricula in Mexico.

We have achieved top honours in the Student Design Competitions during the ACM SIGCHI CHI conferences, reaching second place in San José in 2007 and first place in Firenze, in 2008 .

Our university became the first site in Mexico to hold a special World Usability Day in 2007

\section{THE USALAB EXPERIENCE}

\subsection{Usablity Lab in Universities in Mexico}

We were convinced that we needed a usability lab in order to continue and specialise our work. An initial investigation showed us that the only lab in Mexico developed to usability testing wasn't entirely devoted to it. We decided to create the first usability lab in public universities in the country. Based in the paper entitled "ReUsaLability" [2], in which it described the re-use of equipment from different areas in our university, we finally created our first lab, named UsaLab (from the English 
terms of "Usability Laboratory" and creating a similar concept to those of LavaTap, a well known cleaning company in Mexico). UsaLab featured a Gesell chamber, observation room, user room, video equipment and analysis software from Noldus, as shown in the Figures 1 and 2 below.
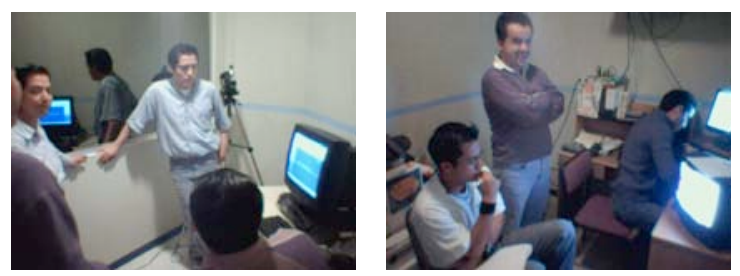

Figures 1 and 2. User room (left) and observation room at UsaLab

UsaLab started teaching and then performing formal usability testing to thesis project being developed at the time by undergraduates. Suddenly it became a requirement to our neighbour Distributed Systems Research Group, which housed the research line of Children's Educational Software Development.

CASI required that all developments being done by the research group included usability testing and recommended a user centered design methodology. Lecturers started teaching the advantages of usability in their projects.

With the help of several research groups related to the area (eg universities of Colima, Yucatán, Tlaxcala, Puebla's UDLA and INAOE, Monterrey's ITESM and Mexico's UNAM), UsaLab started providing services to external academic institutions, reaching al least 120 usability studies made, either as a free service or as part of academic projects with another institution.

\subsection{Services to the Industry}

Being the only usability lab in public universities, we decided to move forward to professionalise our services in order to offer it to the ever-growing software industry in the region and country. As well, as our students would have a better perspective of the real world outside, our researchers would have a chance to create applied research and the university would have additional income from our services.

Along with our university's first software development company, KadaSoftware, we started a period of "evangelisation" in which we sprayed the word on the economical benefits and ways to adapt usability and a user centered design approach on software development through a circuit of conferences, workshops and articles posted in both organisation's web sites. We adopted a different image on our web site, creating a new, more formal industry appearance and got a commercial domain for our site.

UsaLab adopted the Common Industry Format for Usability Test Report v2.0 format for results report on usability testing. Formalised the user recruitment by the use of databases and tried and developed more useful methodologies and techniques. We learned and adopted a business culture without regarding our beloved academic background.

\section{LESSONS LEARNED}

HCI and usability is a hard sell. Still there are few local companies interested and quite few willing to pay for our services. Also, we have been struggling to convert the local market into an industry demanding services. Of course, there have been progresses with more and more companies are requesting quotes, information and willing to learn about the benefits of applied usability.

Our larger customer base has been foreign companies looking to break through international products into the Mexican market. Our decisive advantage has been the local knowledge of the culture, people and tech market. Along with our international partner Mercedes Sánchez Usabilidade from Brazil, we have been mainly developing business proposals for Asian mobile manufacturers clients. They are well versed in the benefits of a market research and usability studies on their products.

Another important issue is to seek partnerships with local research groups within Mexico. Generally, the usability studies requires testing in several locations around Mexico, so we should be better acquainted with more usability labs and professional personnel in the country. We are in talks to develop new labs in host universities and to establish connections with the few ones in the industry.

Our experience has taught us that our best promotion and advertisement are the academic results achieved by our students and faculty in the area. The second and first place in the CHI conferences allowed us to gain a lot of respect and credibility across the industry. Our best propaganda and contacts are being made, oddly, at scientific forums.

Another lesson learned is to find a niche of specialisation. We have gained extensive knowledge developing children learning applications for mobiles devices and both the Motorola Foundation and Palm de Mexico have founded projects as part of their product placement campaign.

\section{FUTURE DIRECTONS}

We are looking to certify our personnel on the Usability Professionals Association, the UPA. We also have been developing the new UsaLab facilities equipped with new state of the art gear, such as cameras for mobile devices, eye tracking and a mobile usability lab.

We will continue our academic programme looking forward to consolidate our place, diversifying our efforts, aiming to another competitions, like the Research Student Competition in ACM or the Imagine Cup from Microsoft.

Constantly looking for new markets, we are in touch with European and Chinese companies interested in Latin America and local knowledge. We are eager to built momentum and establish partnerships with usability labs and to create a larger market for our activities.

\section{ACKNOWLEDGMENTS}

The authors would like to thank our university, the Mexican SIGCHI and our partners, especially KadaSoftware, Mercedes Sánchez and Dr Christian Sturm for their continued efforts and support.

\section{REFERENCES}

[1] Moreno Rocha M A. Adding Human Computer Interaction Studies into Informatics and Computing Engineering Bachelor Degrees in Latin America. In Proceedings of the Conference on Human Factors in Computing Systems (CHI 2001), Seattle, USA, ACM Press, New York, NY, 2001

[2] Moreno Rocha M A. ReUsaLability, Propuesta de Creación de un Laboratorio de Usabilidad: UsaLab. URL http://mixtli.utm.mx/ usalab/Archivos/Usalab.pdf 